Check for updates

Cite this: RSC Adv., 2020, 10, 3705

Received 11th October 2019

DOI: $10.1039 / c 9 r a 08274 c$

rsc.li/rsc-advances
Accepted 10th December 2019

\section{A magnetic nanoscale metal-organic framework (MNMOF) as a viable fluorescence quencher material for ssDNA and for the detection of mercury ions via a novel quenching-quenching mechanism $\uparrow$}

\begin{abstract}
Muppidathi Marieeswaran and Perumal Panneerselvam (iD) *
A novel fluorescent biosensor has been designed and synthesized comprising a magnetic nanoscale metalorganic framework (MNMOF) functionalized with fluorescein amidite (FAM)-labeled ssDNA. It exhibits good sensitivity and selectivity for $\mathrm{Hg}(॥)$ cations over other co-existing metal ions. MNMOF was fabricated by a one-pot synthetic method and it was successfully characterized with various techniques such as UVvisible spectroscopy, fluorescence spectroscopy, Fourier-transform infrared (FT-IR) spectrometry, X-ray diffraction (XRD), scanning electron microscopy (SEM), transmission electron microscopy (TEM) and $X$ ray photoelectron spectroscopy (XPS). The FAM-labeled ssDNA was adsorbed onto the surface of MNMOF through $\pi-\pi$ stacking and electrostatic interactions, which resulted in the partial quenching of its fluorescence intensity (65\%). Upon the subsequent addition of $\mathrm{Hg}($ (I) ions, the fluorescence intensity was further quenched at $52 \%$, due to the re-adsorption of dsDNA onto the surface of MNMOF. Thus, the FAM-labeled ssDNA showed a drastic decrease in fluorescence intensity with $\mathrm{Hg}(I)$. This quenchingquenching mechanism led to a linear response in the fluorescence intensity to $\mathrm{Hg}\left(\right.$ (I) concentration $\left(R^{2}=\right.$ 0.934) with a low detection limit of $8 \mathrm{nM}$. The specific merits of MNMOF make it an ideal platform for mercury sensor applications.
\end{abstract}

\section{Introduction}

The development of biosensors based on highly sensitive fluorescent probes for the selective detection of heavy metals and transition metal ions is difficult, but it has been an area of focus for the scientific community in the past few years due to concerns regarding human health and environmental safety. Mercury ions have been considered as one of the most toxic heavy metals, causing Minamata diseases, neurodevelopment disorders, subclinical brain dysfunction, kidney failure, and cancer. Furthermore, the World Health Organization (WHO) has set a permitted limit for $\mathrm{Hg}$ (II) in drinking water of $30 \mathrm{nM}$. Therefore, environmental monitoring of aqueous $\mathrm{Hg}$ (II) is in increasing demand. ${ }^{1,2}$

So far, many analytical methods have been proposed for the detection of $\mathrm{Hg}$ (II) such as atomic absorption spectroscopy, inductively coupled plasma mass/atomic emission spectrometry, ultraviolet (UV)-visible spectroscopy, high-performance

Department of Chemistry, SRM Institute of Science and Technology, Kattankulathur, 603 203, Tamil Nadu, India. E-mail: panneerp1@srmist.edu.in; panneerchem82@ gmail.com; Tel: +919688538842

$\dagger$ Electronic supplementary information (ESI) available. See DOI: $10.1039 / \mathrm{c} 9 \mathrm{ra} 08274 \mathrm{c}$ liquid chromatography, colorimetric and electrochemical sensors, and so on. ${ }^{3-8}$ The existing techniques have limitations: they can be time-consuming, and require high-cost instruments and highly skilled personnel. To overcome the drawbacks, nucleic acid-based fluorescent sensors have been proposed.9-12 Among the existing techniques, fluorescence-based nucleic acid such as fluorescein amidite (FAM), tetramethylrhodamine (TAMRA), and Cys-labeled oligonucleotides for the precise detection of mercury. Recently, numerous fluorescence quencher materials such as graphene oxide (GO), nanoparticles (NPs), carbon nanotubes (CNTs), $\mathrm{MoS}_{2}$ and polydopamine nanotubes ${ }^{13-18}$ have been used more frequently for the detection of mercury ions.

The progressive development of synthetic processes for the production of metal-organic frameworks (MOFs) has been reported as well as their unique characteristics linked to fascinating properties such as excellent performance-selectable composition, high surface area, tunable porosity, high electrical conductivity, and optical properties. ${ }^{19-22}$ As expected, nanoscale metal-organic frameworks (NMOFs) have promising properties, such as high functionality, strong bio-affinity, and high stability. NMOFs have shown potential applications in versatile fields, such as catalysis, drug delivery, adsorption, gas storage, ( $\mathrm{T}-\mathrm{Hg}(\mathrm{II})-\mathrm{T})$ sensors have been described using various dyes 
photocatalysis, and sensing applications. ${ }^{24-27}$ Moreover, various synthetic routes have been employed to produce NMOFs, such as nanoscale precipitation, and solvothermal, surfactanttemplate, and reverse microemulsion methods. ${ }^{23}$

Very recently, some NMOFs have been designed for detection based on the effective fluorescence sensing platform of small molecules and DNA. Studies have been documented with MOFs including UiO-66- $\mathrm{NH}_{2}, \mathrm{MIL}-88 \mathrm{~B}, \mathrm{H} 2 \mathrm{dtoaCu}$, and MIL-101, which show tremendous abilities to bind with dye-labeled DNA, quenching its fluorescence intensity. ${ }^{28-30}$ NMOFs bind to dye-labeled ssDNA through electrostatic, $\pi-\pi$ stacking, and/or hydrogen-bonding interactions. Therefore, the fluorescence is quenched by photoinduced electron transfer (PET) and fluorescence resonance energy transfer (FRET) mechanisms. ${ }^{31}$

$\mathrm{Fe}_{3} \mathrm{O}_{4}$ NPs have received great attention because of their magnetic and adsorption properties. ${ }^{32}$ Yuling and co-workers fabricated $\mathrm{Fe}_{3} \mathrm{O}_{4} @$ MOFs for photocatalytic contaminant removal and drug delivery. ${ }^{33}$ Yan and co-workers synthesized $\mathrm{Fe}_{3} \mathrm{O}_{4} @ M O F$ for analytical applications via magnetic solidphase extraction. ${ }^{34}$ Zhang and co-workers designed and synthesized $\mathrm{Fe}_{3} \mathrm{O}_{4} @ \mathrm{MOF}$ (MIL-53(Fe)) for the degradation of organic pollutants. $^{35}$

In this work, we focused our efforts on FAM-labeled ssDNA adsorbed on the surface of a magnetic NMOF (MNMOF)-based biosensing platform to increase the sensitivity for $\mathrm{Hg}$ (II) detection. The FAM-labeled ssDNA could be adsorbed on the surface of MNMOF through $\pi-\pi$ stacking interactions, which led to a quenching of the fluorescence emission to $65 \%$ of the initial level. The addition of $\mathrm{Hg}$ (II) further enhanced the quenching of the fluorescence at $52 \%$, as a result of $\mathrm{Fe}^{3+}$ inorganic metal nodes present on the surface of MNMOF. To the best of our knowledge, this is the first report of a fluorescence quenchingquenching mechanism based on an MNMOF for an environmental application. The strategy gave a good analytical response in the determination of mercury. Furthermore, this MNMOF has a high quenching ability compared to those of other nano quencher materials.

\section{Methods}

\section{Materials and measurements}

All chemicals were purchased from Sigma Aldrich, Avra and SRL chemicals and were of analytical grade. These included ferric chloride $\left(\mathrm{FeCl}_{3}\right)$, 2-aminoterephthalic acid, dimethylformamide (DMF), methanol, mercury nitrate, cadmium chloride, ferric chloride, magnesium chloride, silver chloride and other standards. Solvents were used without any further purification. Aqueous solutions were prepared from Milli-Q water. The buffer was prepared from $50 \mathrm{mM}$ Tris- $\mathrm{HCl}, \mathrm{NaCl}$ (50 mM) and $\mathrm{MgCl}_{2}$ $(5 \mathrm{mM})(\mathrm{pH}=7.4)$. The stock solution of DNA was prepared by using Tris-HCl buffer. The synthesized DNA was purchased from Integrated DNA Technologies (IDT, Avantor, India) and the DNA probe sequence was $5^{\prime}$-56-FAM-ATT TGT TTT GTT TCC CCT TTC TTC TTT TCT TTT- $3^{\prime}$

Fluorescence spectroscopy was performed on a HORIBA scientific spectrofluorometer using cuvettes of $1.0 \mathrm{~cm}$ path length with a xenon lamp excitation source and an excitation wavelength of $490 \mathrm{~nm}$. The morphology and crystalline structure of MNMOF were evaluated using scanning electron microscopy (SEM, FEI Quanta FEG 200) and high-resolution transmission electron microscopy (HR-TEM, JEOL, JEM, Fb2000). X-ray diffraction (XRD) was performed using a PAN analytical X'pert pro X-ray diffractometer with $\mathrm{CuK} \alpha$ radiation. The bonding information studies were performed on an Agilent Technologies FTIR spectrometer (USA); Fourier transform infrared (FT-IR) spectra were recorded in attenuated total reflectance (ATR) mode.

\section{Synthesis of NMOF $\mathrm{NH}_{2}$-MIL-101(Fe)}

$\mathrm{NH}_{2}$-MIL-101(Fe) was prepared using a previously reported method with some modifications. ${ }^{36}$ Typically, $1.6 \mathrm{~g}$ of $\mathrm{FeCl}_{3}$ and $1.4 \mathrm{~g}$ of 2-aminoterephthalic acid were dissolved in DMF/ methanol with stirring at ambient temperature. The resulting homogenous solution was transferred to a $100 \mathrm{~mL}$ Teflon-lined autoclave and then heated at $150{ }^{\circ} \mathrm{C}$ for $12 \mathrm{~h}$. After cooling down to room temperature, the brown precipitate was separated by centrifugation, washed with DMF and methanol and dried in a hot-air oven at $60{ }^{\circ} \mathrm{C}$ overnight.

\section{Synthesis of $\mathrm{Fe}_{3} \mathrm{O}_{4}$ nanoparticles}

$\mathrm{Fe}_{3} \mathrm{O}_{4}$ NPs were prepared using a simple hydrothermal technique based on the previously reported literature. ${ }^{21}$ In brief, $5 \mathrm{mmol}$ of $\mathrm{FeCl}_{3} \cdot 6 \mathrm{H}_{2} \mathrm{O}$ and $43.8 \mathrm{mmol}$ of $\mathrm{CH}_{3} \mathrm{COONa}$ were dissolved in $40 \mathrm{~mL}$ of ethylene glycol and magnetically stirred for 45 minutes. The obtained solution was transferred into a $100 \mathrm{~mL}$ Teflon-lined stainless-steel autoclave and kept in a muffle furnace at $200{ }^{\circ} \mathrm{C}$ for $10 \mathrm{~h}$. After cooling to room temperature, the obtained black precipitate was separated, washed several times with distilled water and ethanol and finally dried in ambient conditions.

\section{Synthesis of MNMOF}

MNMOF was synthesized via a one-pot method. $\mathrm{Fe}_{3} \mathrm{O}_{4}$ nanospheres $(0.020 \mathrm{~g}), \mathrm{FeCl}_{3} \cdot 6 \mathrm{H}_{2} \mathrm{O}(1 \mathrm{mmol})$ and 2-aminoterephthalic acid $(1 \mathrm{mmol})$ were added into $15 \mathrm{~mL}$ of DMF solution and ultrasonicated for about $15 \mathrm{~min}$. The mixture was then transferred into a pressure tube with a volume capacity of $60 \mathrm{~mL}$ and heated at $130{ }^{\circ} \mathrm{C}$ for 2 days. After the heat treatment, the product was collected by centrifugation at $6000 \mathrm{rpm}$. To remove excess solvent and unreacted precursor, the obtained powder was dried under vacuum at $60{ }^{\circ} \mathrm{C}$ for $12 \mathrm{~h}$.

\section{Analysis of environmental water samples}

The sample tap water was collected from our laboratory. This environmental sample was filtered through a membrane to remove any insoluble impurities. Aliquots of the environmental water sample were spiked with a stock solution of $\mathrm{Hg}$ (II) and diluted with Tris-HCl buffer. Then fluorescence measurements were performed. 


\section{Results and discussion}

Structural information from powder X-ray diffraction (PXRD) studies of the synthesized materials, namely $\mathrm{Fe}_{3} \mathrm{O}_{4} \mathrm{NPs}, \mathrm{NH}_{2}$ MIL-101(Fe) (NMOF), and $\mathrm{NH}_{2}-\mathrm{MIL}-101$ (Fe)@Fe $\mathrm{O}_{4}$ (MNMOF), are represented in Fig. 1a. The NMOF characteristic diffraction peaks exist at low $2 \theta$ values of $9.04^{\circ}, 10.30^{\circ}$, and $13.10^{\circ}$, consistent with previous literature reports. Furthermore, the formation of $\mathrm{Fe}_{3} \mathrm{O}_{4}$ NPs is demonstrated from the crystalline nature and diffraction peaks at $30.2^{\circ}, 35.5^{\circ}, 43.1^{\circ}, 57.1^{\circ}$, and $62.7^{\circ}$ corresponding to the (220), (311), (200), (511), and 400, planes of $\mathrm{Fe}_{3} \mathrm{O}_{4}$, respectively. ${ }^{37}$ Finally, we confirmed the formation of MNMOF from the combination of $\mathrm{Fe}_{3} \mathrm{O}_{4}$ NPs and NMOF diffraction peaks found at $10.30^{\circ}, 30.2^{\circ}, 35.5^{\circ}, 43.1^{\circ}$, $57.1^{\circ}$, and $62.7^{\circ}$. These data indicate that the $\mathrm{Fe}_{3} \mathrm{O}_{4}$ NPs are incorporated to give MNMOF without any disturbance to the NMOF crystalline nature. Moreover, MNMOF does not show any detectable crystalline impurities or peak shifts indicating its high purity. Fig. 1b illustrates the FT-IR spectra of $\mathrm{Fe}_{3} \mathrm{O}_{4}$ NPs, NMOF and MNMOF to clarify the chemical bonding interactions. As displayed in Fig. 1b, the FT-IR spectrum of NMOF shows two major functional groups, namely amine $(\mathrm{N}-\mathrm{H})$ and carbonyl $(\mathrm{C}=\mathrm{O})$, through sharp absorption peaks at $3477 \mathrm{~cm}^{-1}$ $\left(\mathrm{N}-\mathrm{H}\right.$ str), and $1679 \mathrm{~cm}^{-1}(\mathrm{C}=\mathrm{O})$ respectively. The $\mathrm{Fe}_{3} \mathrm{O}_{4} \mathrm{NPs}$ have strong peaks at around $669 \mathrm{~cm}^{-1}$, corresponding to the $\mathrm{Fe}-$ O band. Moreover, the MNMOF spectrum shows a combination of the peaks of the parent materials, with a broad peak at $3477 \mathrm{~cm}^{-1}$ attributed to the stretching frequency of $\mathrm{N}-\mathrm{H}$, and peaks at 1568 and $1398 \mathrm{~cm}^{-1}$ ascribed to the symmetric and asymmetric frequency of the carbonyl group. These FT-IR results confirm the formation of MNMOF.

The hysteresis loops and magnetic properties of $\mathrm{Fe}_{3} \mathrm{O}_{4} \mathrm{NPs}$, NMOF and MNMOF were investigated. Fig. 2 (inset) shows that the presence of $\mathrm{Fe}_{3} \mathrm{O}_{4}$ NPs leads to the facile separation of MNMOF from the sensing probe by the application of an external magnet. In this analysis, the MNMOF magnetization curve exhibits a paramagnetic nature (Fig. 2). The pristine $\mathrm{Fe}_{3} \mathrm{O}_{4}$
NPs have a high magnetization value of $1.6 \mathrm{emu}$ compared to that of NMOF $\left(4.2933 \times 10^{-3} \mathrm{emu}\right)$. MNMOF shows a saturation magnetization value that is estimated to be $79.65 \times 10^{-3} \mathrm{emu}$ (see the enlarged magnetic curve in Fig. 2), indicating an increase in magnetization and good super magnetism compared to those of NMOF. As shown in the inset to Fig. 2, MNMOF can be easily separated from solution using an externally applied magnet.

Extensive morphological studies were conducted to better understand the synthesized $\mathrm{Fe}_{3} \mathrm{O}_{4}$ NPs, NMOF and MNMOF as shown in Fig. 3, S1, S2, and S9. $\dagger$ Fig. S1 $\uparrow$ shows that the pristine $\mathrm{Fe}_{3} \mathrm{O}_{4}$ NPs exist as spheres with uniform dispersion. NMOF $\left(\right.$ MIL-101- $\mathrm{NH}_{2}$ ) displays hexagonal rod-like structures (Fig. S2 $\dagger$ ), and its surface is smooth with no apparent isolated particles. Fig. 3a and b show SEM images which confirm the formation of MNMOF. It is constructed from $\mathrm{Fe}_{3} \mathrm{O}_{4}$ NPs and NMOF and the particles are uniformly dispersed. In addition, energy-dispersive $\mathrm{X}$-ray spectroscopy (EDX) analysis indicates that MNMOF consists of $\mathrm{Fe}, \mathrm{C}, \mathrm{O}$, and $\mathrm{N}$ elements, and that $\mathrm{Fe}_{3} \mathrm{O}_{4}$ NPs are anchored on the surface of MNMOF (Fig. S3†). Furthermore, the EDS elemental mapping also confirms that $\mathrm{Fe}, \mathrm{C}, \mathrm{O}$, and $\mathrm{N}$ elements are distributed on the surface of MNMOF (Fig. 3c). Additionally, HR-TEM microscopy images evidently show $\mathrm{Fe}_{3} \mathrm{O}_{4}$ NPs anchored on the surface of MNMOF rods (Fig. S3†). The HR-TEM microscopy images of MNMOF are displayed in Fig. 4. As can be seen in Fig. 4, $\mathrm{Fe}_{3} \mathrm{O}_{4}$ NPs are anchored on the surface of the MNMOF rods with a narrow size distribution.

The chemical analysis and bonding configuration of MNMOF were investigated by X-ray photoelectron spectroscopy (XPS). The survey spectrum (Fig. 5a) shows C 1s, N 1s, O 1s, Fe 2p and Fe 2s peaks at 284.9, 399.1, 532.09, 726.5 and $894.46 \mathrm{eV}$, respectively. ${ }^{39}$ Fig. 5b shows the high-resolution XPS spectrum of $\mathrm{C}$ 1s, which emphasizes surface components, such as the benzoic and carboxylate groups of organic linkers with binding energies of 284.8 and $289.4 \mathrm{eV}$, respectively. ${ }^{\mathbf{4 0 , 4 1}}$ The O 1 s spectrum can be fitted by two peaks at binding energies of around 532.2 and $531.2 \mathrm{eV}$, which can be attributed to the organic
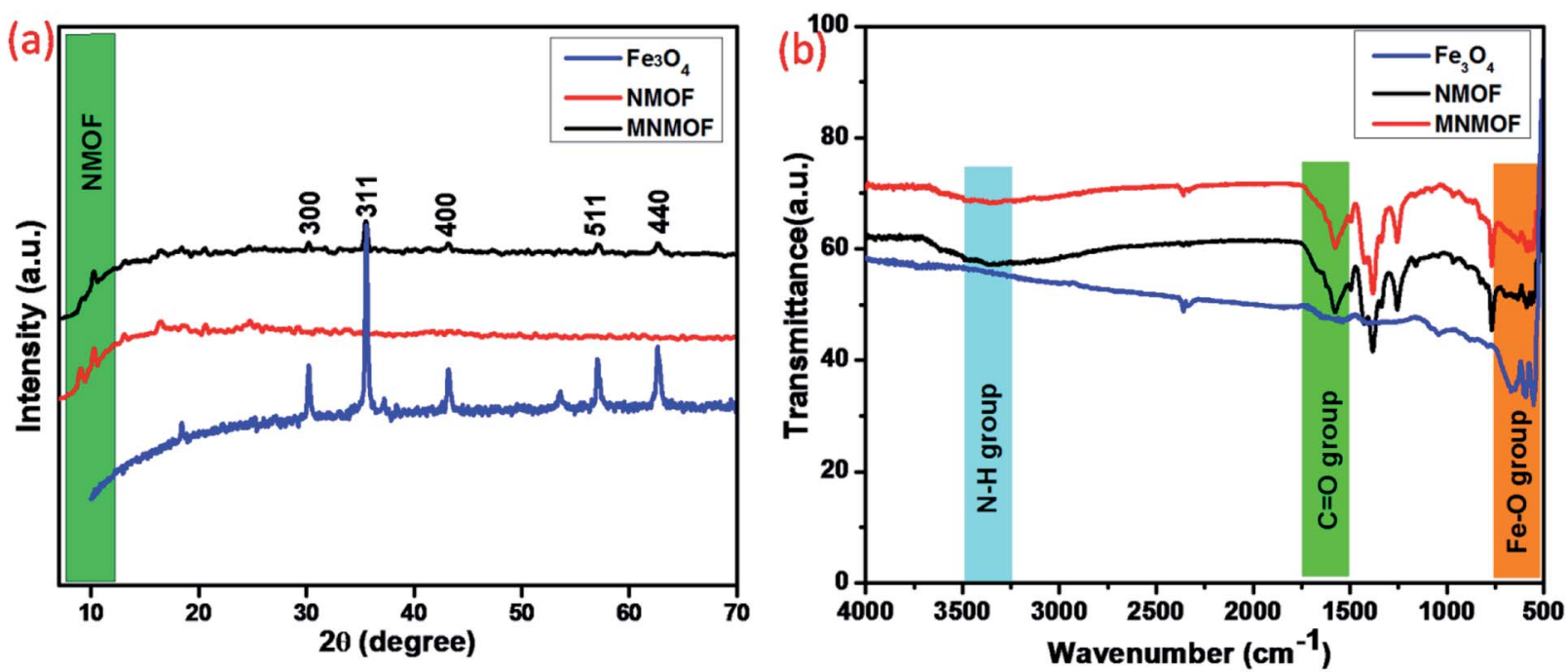

Fig. 1 (a) PXRD patterns of $\mathrm{Fe}_{3} \mathrm{O}_{4}$ NPs, NMOF and MNMOF, (b) FT-IR spectra of $\mathrm{Fe}_{3} \mathrm{O}_{4} \mathrm{NPs}$, NMOF and MNMOF. 


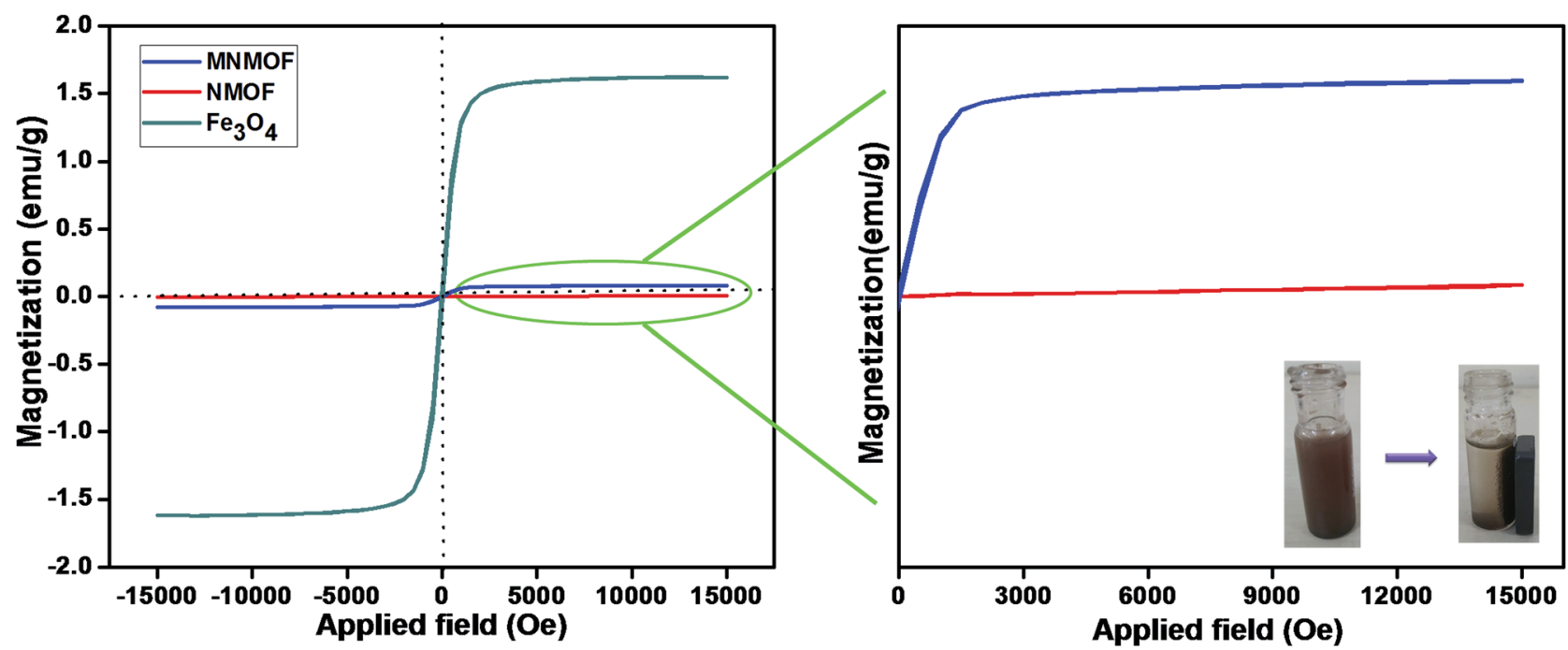

Fig. 2 Hysteresis loops of synthesized $\mathrm{Fe}_{3} \mathrm{O}_{4} \mathrm{NPs}, \mathrm{NMOF}$ and MNMOF. The inset shows digital images for MNMOF before and after magnetic separation achieved using an external magnetic field.

linker of carboxylate groups and $\mathrm{Fe}-\mathrm{O}$ bonds in the $\mathrm{Fe}_{3} \mathrm{O}_{4} \mathrm{NPs}$ (Fig. 5c). Fig. 5e shows the high-resolution XPS spectrum of Fe 2p. The binding energies of $713.5,725.7$ and $718.02 \mathrm{eV}$ are characteristic of $\mathrm{Fe}^{3+}$ in MNMOF, while the peaks at binding energies of 711.4 and $726.5 \mathrm{eV}$ are typical for magnetite. ${ }^{42}$

Quenching-quenching mechanism of MNMOF in the detection of $\mathrm{Hg}$ (II)

In general, transition-metal ions have intrinsic fluorescence quenching properties. Recently, very few nanomaterials have been used as fluorescence quenchers, including GO, CNTs, gold NPs and $\mathrm{MoS}_{2}$ nanosheets. These nanomaterials have been employed in sensing strategies using fluorescence quenching and recovery of dye-labeled ssDNA. In contrast, MNMOF shows an unusual fluorescence quenching mechanism with FAMssDNA. Here, we explored the analytical performance of the novel fluorescence quenching mechanism represented in Scheme 1. FAM-labeled ssDNA is readily adsorbed to the surface of MNMOF via non-covalent interactions. The fluorescence of the FAM-labeled ssDNA is partially quenched by this interaction with MNMOF. Then, $\mathrm{Hg}$ (II) ions are introduced to the ssDNA and are hybridized to form a duplex dsDNA. After hybridization, the fluorescence of FAM is further quenched because the negatively charged carboxyl and phenolic hydroxyl groups of
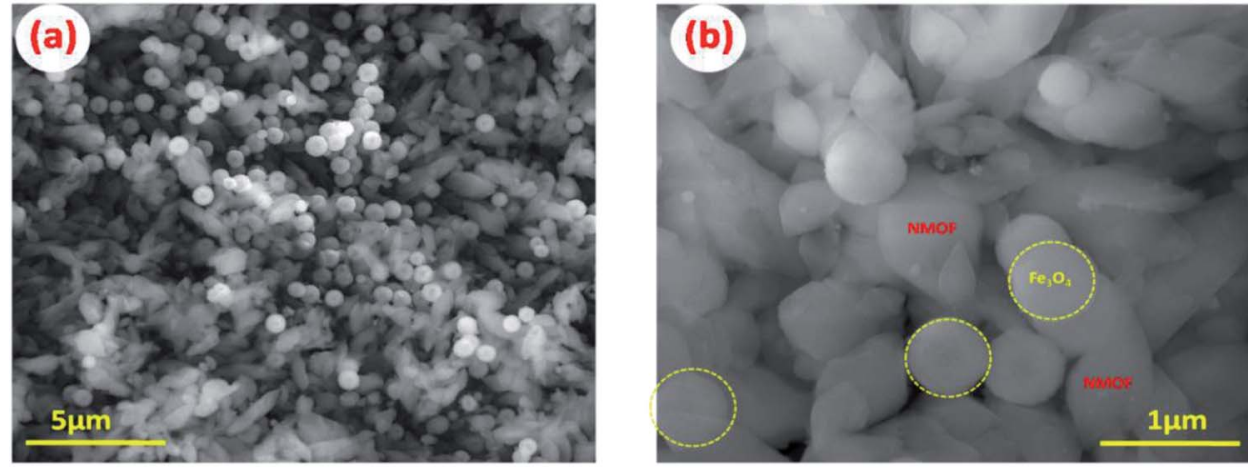

(c)
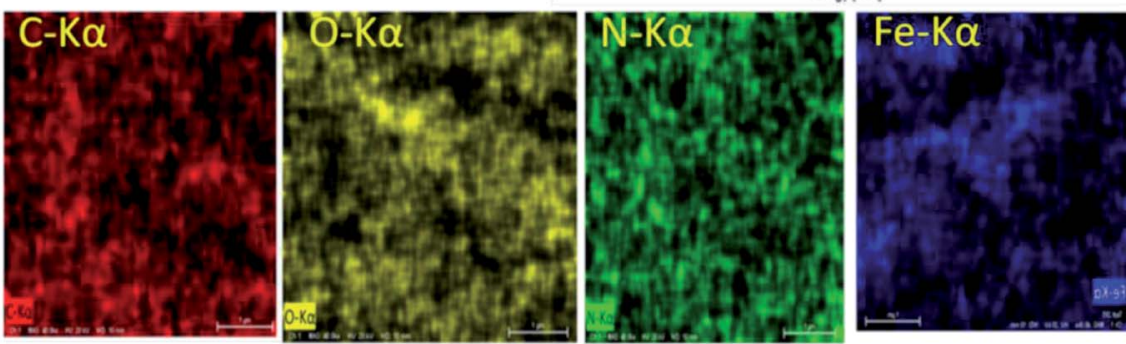

Fig. 3 ( $a$ and b) SEM images of MNMOF, (c) EDS mapping images of MNMOF. 
(a)

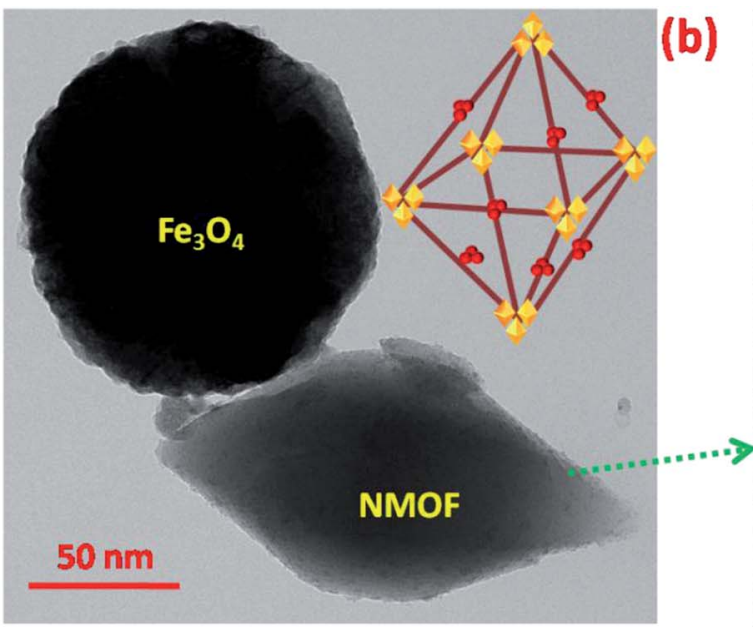

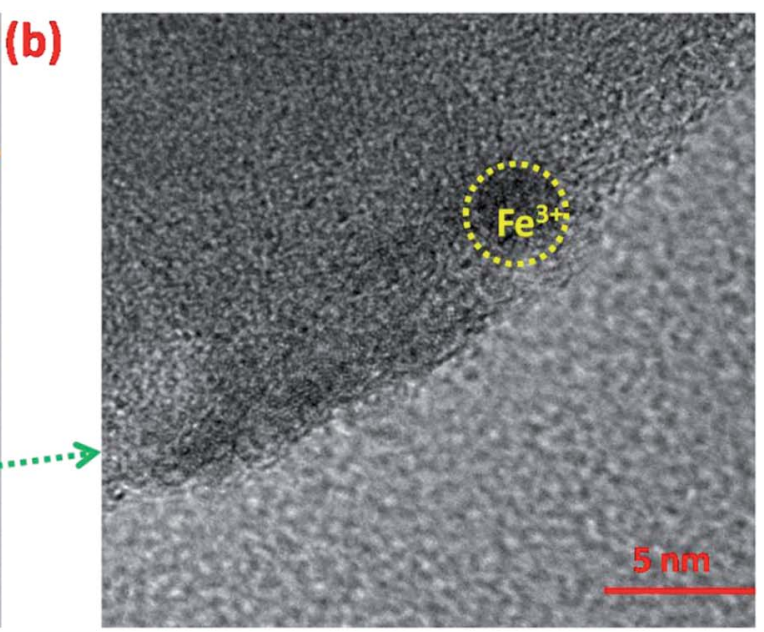

Fig. 4 (a) TEM image of MNMOF, (b) HR-TEM image of MNMOF.

FAM-labeled ssDNA interact with $\mathrm{Fe}^{3+}$ ions on the surface of MNMOF. Further, FAM-dsDNA does not dissociate away from MNMOF, but re-adsorbs on the surface of $\mathrm{Fe}^{3+}$ in MNMOF.

The fluorescence quenching mechanism was investigated by fluorescence and UV-visible spectroscopy. As shown in Fig. 6a, FAM-ssDNA has obvious fluorescence emission at $520 \mathrm{~nm}$ (black line in Fig. 6a). The addition of MNMOF leads to the formation of a hybrid complex that causes partial quenching of the fluorescence intensity (red line in Fig. 6a). Furthermore, the introduction of $\mathrm{Hg}$ (II) ions into the solution containing the hybrid complex results in the further quenching of the fluorescence intensity (blue line in Fig. 6a). The efficient quenching can be attributed to the strong adsorption of FAM-labeled
SSDNA on the surface of MNMOF through $\pi-\pi$ stacking interactions. The fluorescence intensity is decreased due to FRET as displayed in Fig. 6b.

Further, the zeta potentials were measured and used to identify the lattice charges of the synthesized materials, as shown in Fig. 7a. We established that NMOF has a negative surface charge. After incorporation of $\mathrm{Fe}_{3} \mathrm{O}_{4}$ NPs to form MNMOF, the negative zeta potential becomes slightly greater. The UV-visible spectra also provide evidence for a unique quenching mechanism; the results are displayed in Fig. 7b. It can be seen that in the absence of MNMOF, FAM-ssDNA shows an obvious fluorescence emission at $495 \mathrm{~nm}$ in the Tris- $\mathrm{HCl}$ buffer solution. When MNMOF and FAM are combined, the
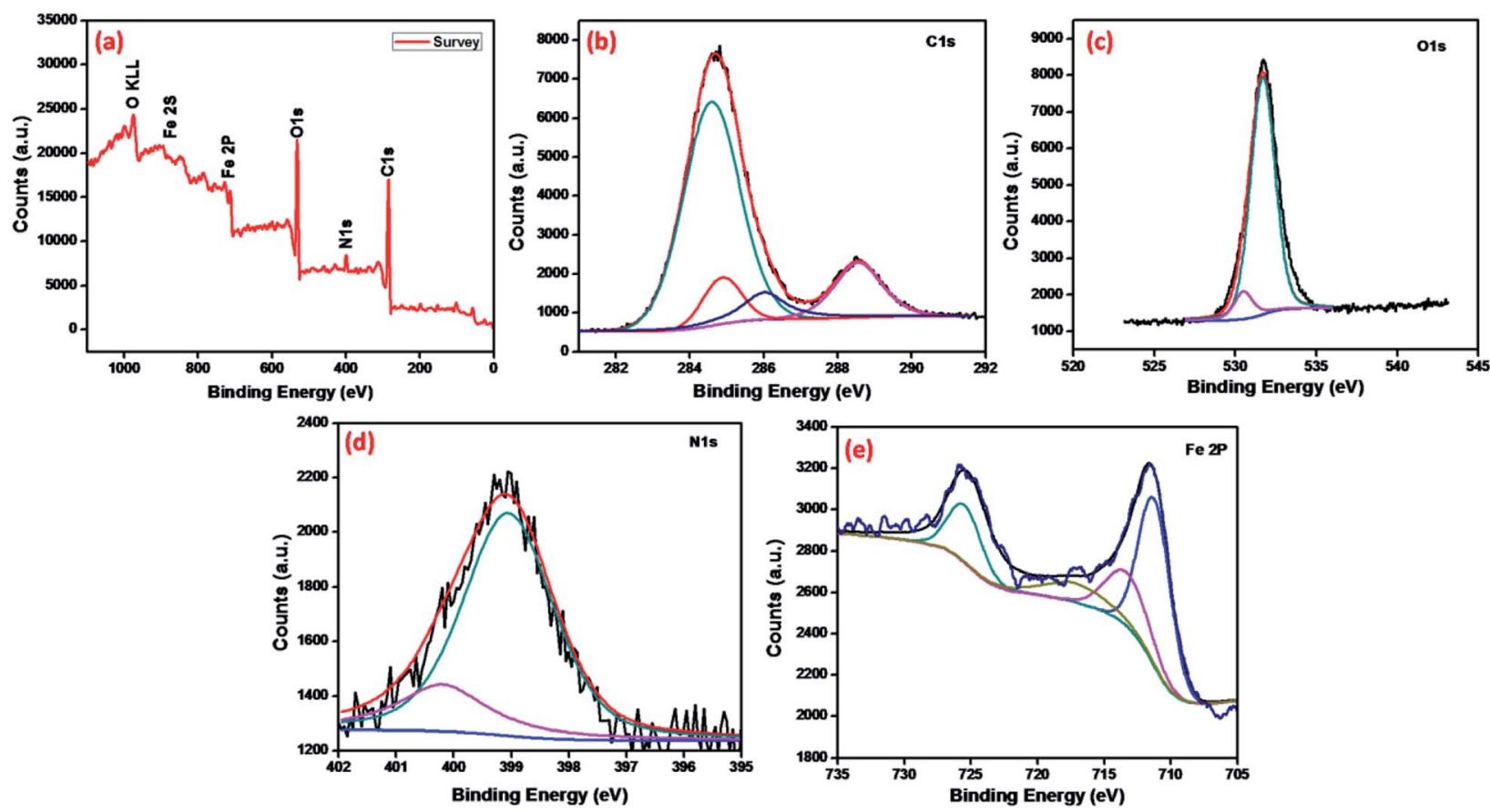

Fig. 5 XPS spectra of MNMOF: (a) survey, (b) C 1s, (c) O 1s, (d) N 1s and (e) Fe 2p. 


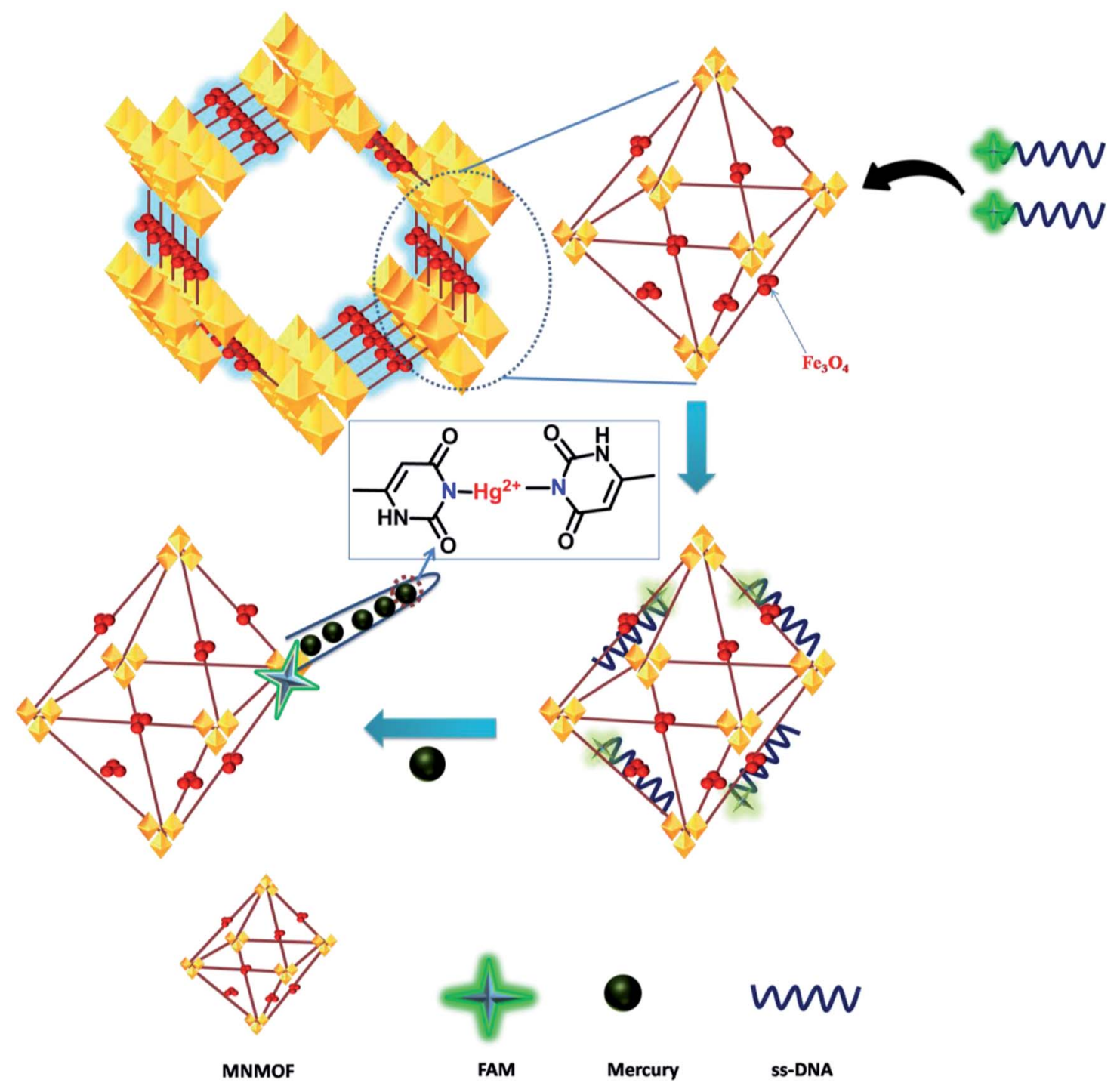

Scheme 1 Schematic illustration of the DNA fluorescence assay with MNMOF as a sensing platform.
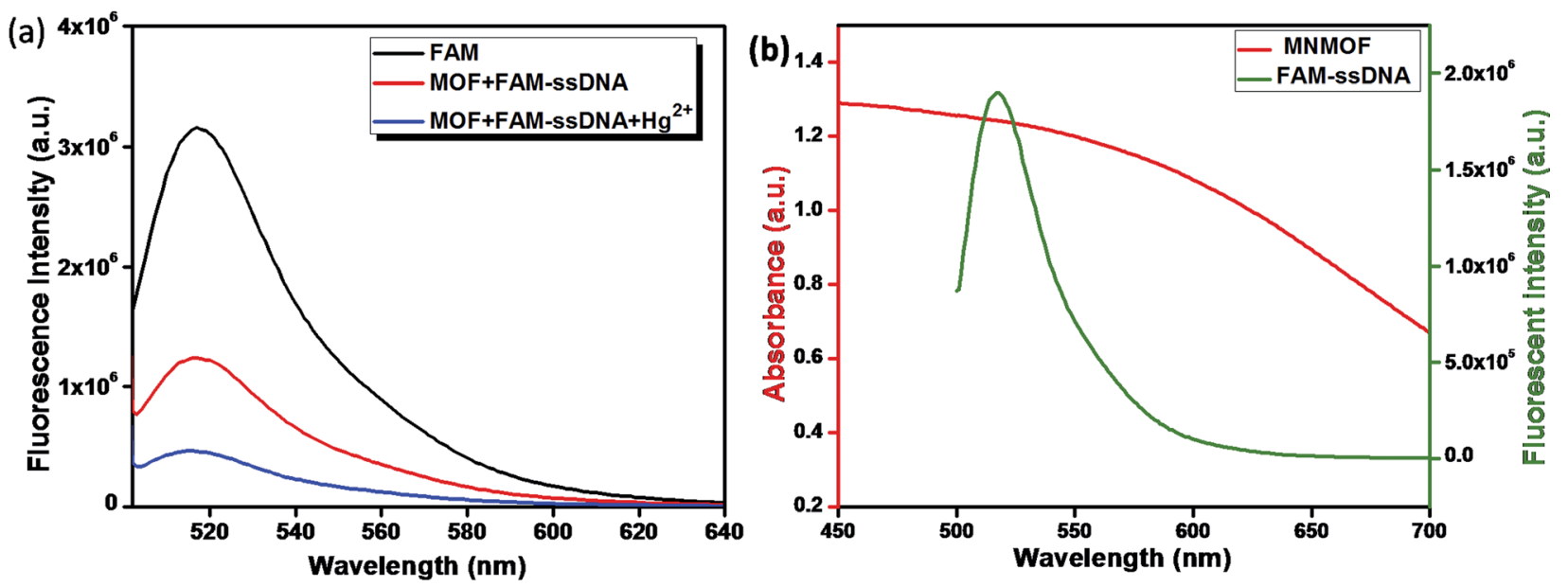

Fig. 6 (a) Schematic illustration of the quenching mechanism and (b) fluorescence spectra of FAM, MNMOF + FAM-sSDNA and MNMOF + FAMssDNA + Hg(॥). 

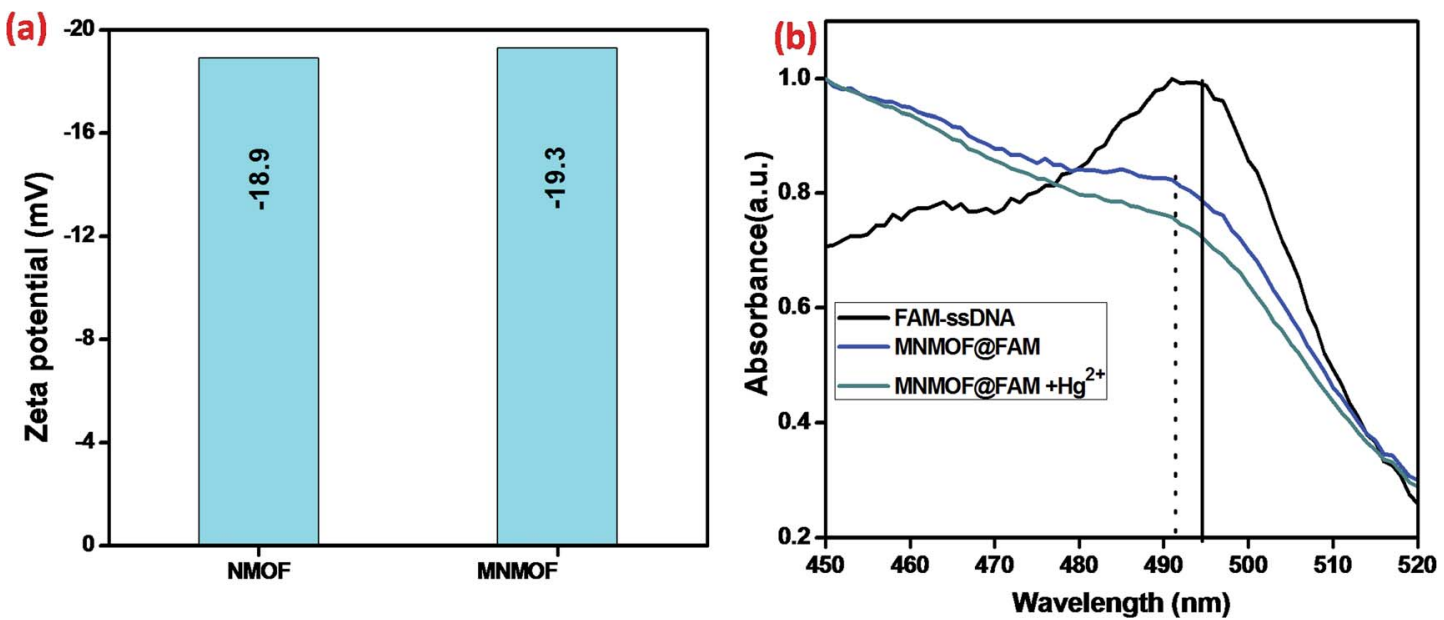

Fig. 7 (a) Zeta-potentials of NMOF and MNMOF, (b) UV-visible spectra of FAM-ssDNA, MNMOF@FAM-ssDNA, and MNMOFaFAM-ssDNA + $\mathrm{Hg}(1)$.

soret band of FAM shifts to a lower wavelength (blue shift). Furthermore, when the $\mathrm{Hg}$ (II) target ions are added into the hybrid complex there is no further shift. As a result, these spectra reveal that FAM-ssDNA does not desorb from the surface of MNMOF when the $\mathrm{Hg}$ (II) ions are added. This is probably since MNMOF contains more uncoordinated $\mathrm{Fe}^{3+}$ ions, which bind the dsDNA.

\section{Fluorescence quenching properties}

MNMOF has excellent fluorescence quenching properties, as shown in Fig. 8. The UV-visible absorption spectrum of FAMssDNA shows an absorption band at $495 \mathrm{~nm}$ (Fig. 8a). Upon the addition of MNMOF, that absorption band shows remarkably hypochromicity; we suggest that this is due to a static interaction between MNMOF and the fluorophore of FAMssDNA. The fluorophore is conjugated with a plane of MNMOF through $\pi-\pi$ interactions and electrostatic interactions. The result is that the absorption band intensity is decreased by MNMOF. The fluorophore of the ssDNA has inherent fluorescence properties and shows a strong emission at $520 \mathrm{~nm}$ (Fig. 8b). By adding MNMOF into the solution containing FAM-ssDNA, this fluorescence intensity is also quenched. Moreover, increasing the concentration (50-350 $\mathrm{ppm}$ ) of MNMOF further quenches the fluorescence intensity, so the fluorescence intensity is inversely proportional to the concentration of MNMOF. Afterwards, we expected the fluorescence intensity to be increased by the introduction of the toxic target ions. In contrast, the intensity of fluorescence emission is quenched even more. In this case, the FAM-ssDNA binds to the target ions to become dsDNA based on $\mathrm{T}-\mathrm{Hg}$ (II)$\mathrm{T}$ interactions.

\section{Optimization and feasibility study}

To optimize the sensing performance of MNMOF, we investigated several influencing factors such as the concentration of MNMOF, quenching time, recovery time and probe
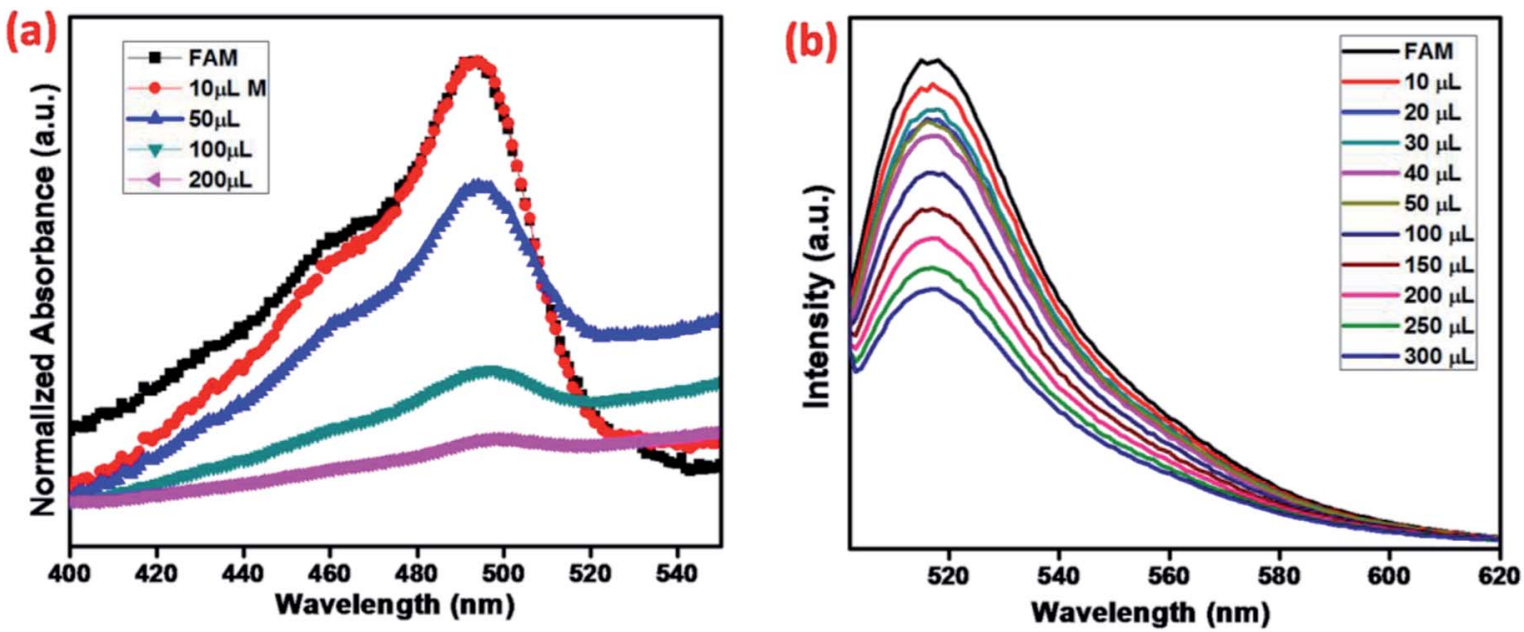

Fig. 8 (a) UV-visible spectra and (b) fluorescence spectra showing the quenching properties of FAM-ssDNA with various amounts of MNMOF. 


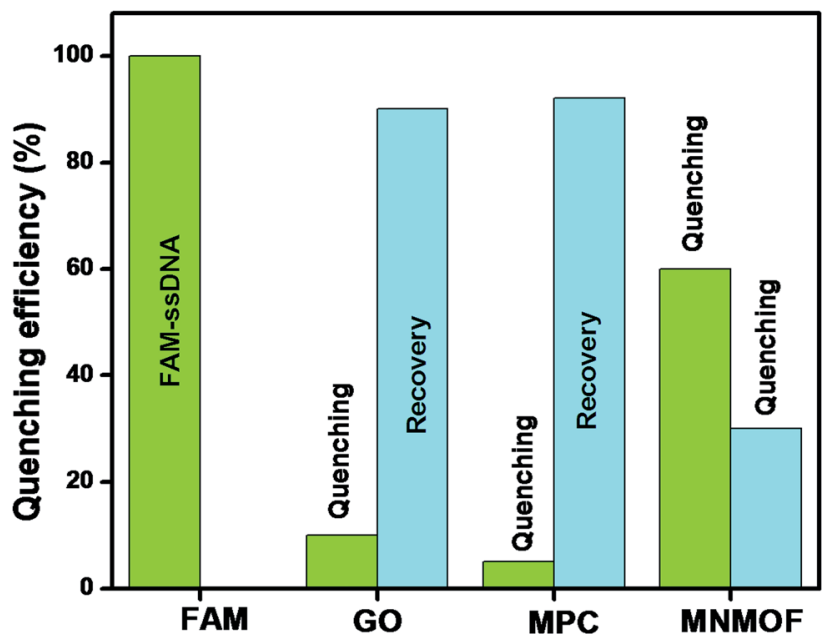

Fig. 9 A comparison of GO, MPC, and MNMOF for quenching and recovery of FAM-ssDNA.

concentration. The concentration of MNMOF was first optimized. As observed in Fig. $\mathrm{S} 3, \uparrow$ the FAM-ssDNA fluorescence intensity gradually decreases with the increase in the concentration of MNMOF. To further understand the adsorption kinetics behavior of the hybridization of FAM-labeled ssDNA and MNMOF, $\mathrm{Hg}$ (II) ions were added during the incubation time (Fig. S5†). In the absence of $\mathrm{Hg}$ (II), the fluorescence signal shows a decrease within 1 minute and it reaches equilibrium at around 7 minutes. The hypochromic curves suggest that MNMOF can adsorb FAM-ssDNA effectively and quickly. After the introduction of $\mathrm{Hg}$ (II) ions, the fluorescence intensity decreases gradually (Fig. $\mathrm{S6} \dagger$ ) and reaches equilibrium at around 6 minutes, suggesting that $\mathrm{Hg}$ (II) binds to the ssDNA to form duplex structures which are effectively re-adsorbed onto MNMOF in a short time. Different concentrations of the probe were added to MNMOF and the results are displayed in Fig. S7†. Then $10 \mu \mathrm{L}$ of MNMOF was used with different concentrations of FAM-SSDNA. MNMOF initially decreases the fluorescence intensity but this increases again with increasing concentrations of FAM-sSDNA. The rapid adsorption and re-adsorption of FAM-ssDNA makes MNMOF promising for the fluorescence sensing of $\mathrm{Hg}$ (II) ions. Fig. 9 shows a comparison of MNMOF with other quencher materials (GO, and MOF-derived $\mathrm{Fe}_{3} \mathrm{O}_{4}$ (MPC)) for the quenching and recovery of fluorescence after the
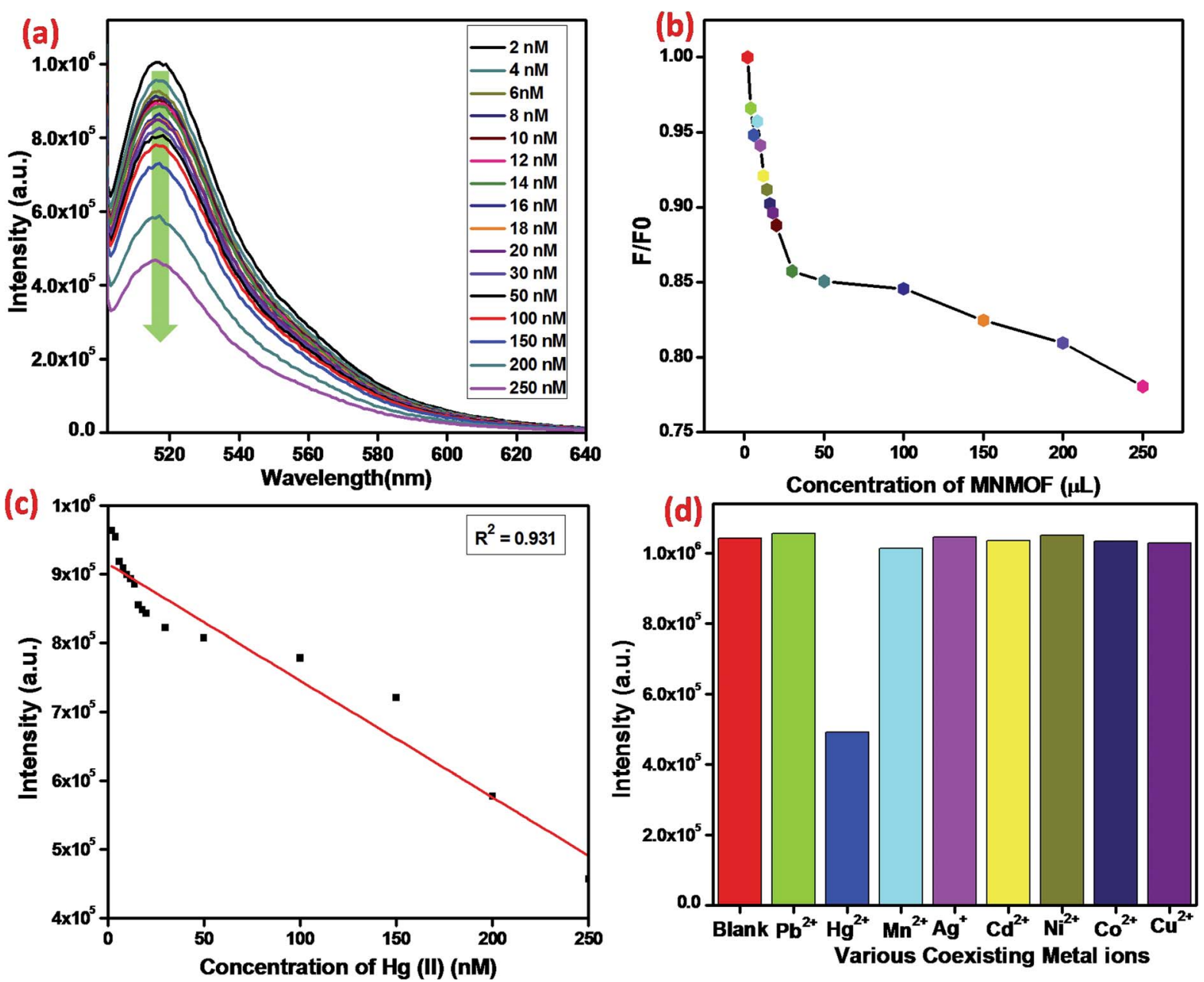

Fig. 10 (a) Fluorescence emission spectra of the FAM-labeled ssDNA and MNMOF with different concentrations of Hg(॥), (b) fluorescence intensity verses $\mathrm{Hg}(॥)$, concentration, (c) the linear relationship between the fluorescence intensity and $\mathrm{Hg}(॥)$ concentration, (D) the selectivity of MNMOF and FAM-ssDNA for $\mathrm{Hg}(I)$ in comparison to that for other co-existing metal ions. 
addition of the quencher and then $\mathrm{Hg}$ (II). MNMOF exhibits a unique quenching mechanism for the detection of $\mathrm{Hg}$ (II) ions.

\section{Detection specificity}

In addition to detection sensitivity, selectivity is a critical factor for evaluating the performance of a proposed sensing system (Fig. 10). Indeed, ssDNA can be used as specific probes in many types of analysis, including for DNA, RNA, protein, small molecules, and other metal ions. The different affinities of MNMOF, ssDNA and their hybrid complex could be used as a universal platform to detect harmful heavy metal ions. Typically, a T-rich ssDNA is designed for the detection of $\mathrm{Hg}$ (II) based on its specific interaction with thymine. When $1 \mathrm{mg} \mathrm{mL}$ of MNMOF is added to $300 \mu \mathrm{L}$ of FAM-ssDNA $(20 \mathrm{~nm})$, adsorption of the FAM-ssDNA occurs on the surface of MNMOF. Therefore, the fluorescence emission is quenched. Moreover, increasing the concentration (50-350 ppm) of MNMOF further quenches the fluorescence intensity (Fig. 10b). The fluorescence intensity is inversely proportional to the concentration of MNMOF. When $\mathrm{Hg}$ (II) is added, the fluorescence intensity is additionally quenched due to the formation of a duplex structure (T-Hg(II)-T) involving the thymine N3 position. Fig. 10c shows the linear relationship between the concentration of $\mathrm{Hg}^{2+}$ ions $(2-20 \mathrm{~nm})$ and the fluorescence intensity; a correlation coefficient value of $R^{2}=0.931$ is obtained and the detection limit is $8 \mathrm{nM}$ according to the $3 \sigma$ method. The specificity of the sensing strategy was investigated by testing the fluorescence response to $\mathrm{Hg}^{2+}$, when the sensor was challenged with other interfering metal ions including $\mathrm{Pb}^{2+}, \mathrm{Mn}^{2+}, \mathrm{Ag}^{+}, \mathrm{Cd}^{2+}, \mathrm{Ni}^{2+}$, $\mathrm{Co}^{2+}, \mathrm{Cu}^{2+}$. As shown in Fig. 10d, the fluorescence intensity is lower for $\mathrm{Hg}^{2+}$ compared with that for interferent ions; these results reveal excellent selectivity for $\mathrm{Hg}^{2+}$ ions. Thus, T-rich ssDNA gives specific detection of $\mathrm{Hg}^{2+}$ based on its specific interaction with MNMOF.

\section{Real sample analysis}

To explore the practical application of the proposed sensor, it was used for the determination of $\mathrm{Hg}$ (II) in a water sample. The water sample was filtered several times before use. Different concentrations of $\mathrm{Hg}$ (II) $(0,5,10$ and $20 \mathrm{nM})$ were spiked into the water sample and analyzed; the results are summarized in Table 1. It can be noted that the proposed biosensor shows satisfactory results and recovery values of around $70 \%$. The findings clearly suggest that our sensing system could be

Table 1 Determination of $\mathrm{Hg}(॥)$ in environmental water samples using the proposed method

\begin{tabular}{llll}
\hline $\begin{array}{l}\text { Spiked }(\mathrm{Hg}(\mathrm{II})) \\
(\mathrm{nM})\end{array}$ & Found (mean $\pm \mathrm{SD})(\mathrm{nM})$ & $\begin{array}{l}\text { Detected } \\
(\mathrm{nM})\end{array}$ & Recovery \\
\hline 0 & - & - & - \\
5 & $3.5 \pm 0.25$ & 3.5 & 70 \\
10 & $7.5 \pm 0.15$ & 7.5 & 70.5 \\
20 & $14 \pm 0.05$ & 14 & 70
\end{tabular}

successfully applied to the analysis of $\mathrm{Hg}$ (II) in real environmental samples.

\section{Conclusion}

In summary, we describe a nucleic acid-based fluorescent biosensor platform that was constructed using FAM-ssDNA with a magnetic nanoscale metal-organic framework (MNMOF) MNMOF was fabricated by a one-pot synthetic method. This effective fluorescence biosensor could be used for the selective detection of $\mathrm{Hg}$ (II) ions over other co-existing metal ions. Noncovalent interactions were employed to adsorb FAM-labeled sSDNA onto the surface of MNMOF and the fluorescence emission was partially quenched. Subsequently, when the $\mathrm{Hg}$ (II) ions were introduced, the fluorescence emission was quenched further at $52 \%$, due to the presence of uncoordinated $\mathrm{Fe}^{3+}$ ions on the surface of MNMOF which re-adsorbed the dsDNA formed after hybridization with $\mathrm{Hg}$ (II). The proposed quenchingquenching approach of the described sensor for $\mathrm{Hg}$ (II) gave a good linear response $\left(R^{2}=0.934\right)$ and a detection limit of $8 \mathrm{nM}$ which compare well to the performance of previously reported sensors. MNMOF has been shown to be an excellent sensing platform, which will stimulate more exciting research; it can potentially be applied to various other heavy metals and biomolecules.

\section{Conflicts of interest}

There are no conflicts to declare.

\section{References}

1 N. Lubick and D. Malakoff, Science, 2013, 341, 1443-1445.

2 U.S. EPA, National Primary Drinking Water Regulations, https://www.epa.gov/ground-water-and-drinking-water/ national-primary-drinking-water-regulations, accessed Mar 10, 2018.

3 Z. H. Lin, G. Zhu, Y. S. Zhou, Y. Yang, P. Bai, J. Chen and Z. L. Wang, Angew. Chem., Int. Ed., 2013, 52, 5065-5069.

4 Q. Pu and Q. Sun, Analyst, 1998, 123, 239.

5 Y. Li, C. Chen, B. Li, J. Sun, J. Wang, Y. Gao, Z. Zhao and Z. Chai, J. Anal. At. Spectrom., 2006, 21, 94-96.

6 M. Krachler, C. Mohl, H. Emons and W. Shotyk, Spectrochim. Acta, Part B, 2002, 57, 1277-1289.

7 P. R. Aranda, P. H. Pacheco, R. A. Olsina, L. D. Martinez and R. A. Gil, J. Anal. At. Spectrom., 2009, 24, 1441-1445.

8 J. Yang, R. Foley and G. K. C. Low, Analyst, 2002, 127, 315318.

9 A. Ravikumar, P. Panneerselvam and K. Radhakrishnan, Microchim. Acta, 2018, 2, 182.

10 R. Saran and J. Liu, Anal. Chem., 2016, 88(7), 4014-4020.

11 S. Singh, K. Mitra, A. Shukla, R. Singh, R. K. Gundampati, N. Misra, P. Maiti and B. Ray, Anal. Chem., 2017, 89, 783-791.

12 B. Li, Y. Du and S. Dong, Anal. Chim. Acta, 2009, 644, 78-82.

13 L. Gao, C. Lian, Y. Zhou, L. Yan, Q. Li, C. Zhang, L. Chen and K. Chen, Biosens. Bioelectron., 2014, 60, 22-29. 
14 H. G. Sudibya, Q. He, H. Zhang and P. Chen, ACS Nano, 2011, 5, 1990-1994.

15 C. C. Huang, Z. Yang, K. H. Lee and H. T. Chang, Angew. Chem., 2007, 119, 6948-6952.

16 Z. Libing, L. Tao, L. Bingling, L. Jing and W. Erkang, Chem. Commun., 2010, 46, 1476-1478.

17 A. Ravikumar, P. Panneerselvam, K. Radhakrishnan, C. A. Anand Babu and S. Sivanesan, Appl. Surf. Sci., 2018, 449, 31-38.

18 A. Ravikumar and P. Panneerselvam, Analyst, 2018, 143, 2623-2631.

19 C. He, D. Liu and W. Lin, Chem. Rev., 2015, 115, 1107911108.

20 J. M. Fang, F. Leng, X. J. Zhao, X. L. Hu and Y. F. Li, Analyst, 2014, 139, 801-806.

21 P. Karthick, A. Pandikumar, M. Preeyanghaa, M. Kowsalya and B. Neppolian, Microchim. Acta, 2017, 184(7), 2265-2273.

22 P. L. William, M. Soumya, D. R. Nathan, V. D. Aamod, L. Jing and K. G. Sujit, Chem. Soc. Rev., 2017, 46, 3242-3285.

23 W. Morris, W. E. Briley, E. Auyeung, M. D. Cabezas and C. A. Mirkin, J. Am. Chem. Soc., 2014, 136, 7261-7264.

24 R. J. Della, D. Liu and W. Lin, Acc. Chem. Res., 2011, 44, 957968.

25 Y. A. Li, C. W. Zhao, N. X. Zhu, Q. K. Liu, G. J. Chen, J. B. Liu, X. D. Zhao, J. P. Ma, S. Zhang and Y. B. Dong, Chem. Commun., 2015, 51, 17672-17675.

26 X. Wei, L. Zheng, F. Luo, Z. Lin, L. Guo, B. Qiu and G. Chen, J. Mater. Chem. B, 2013, 1, 1812-1817.

27 H. T. Zhang, J. W. Zhang, G. Huang, Z. Y. Du and L. H. Jiang, Chem. Commun., 2014, 50, 12069-12072.
28 J. F. Guo, R. M. Fang, C. Z. Huang and Y. F. Li, RSC Adv., 2015, 5, 46301-46306.

29 J. Tian, Q. Liu, J. Shi, J. Hu, A. M. Asiri, X. Sun and Y. He, Biosens. Bioelectron., 2015, 71, 1-6.

30 A. Ravikumar, P. Panneerselvam and N. Morad, ACS Appl. Mater. Interfaces, 2018, 10, 20550-20558.

31 M. Marieeswaran, P. Panneerselvam, A. Ravikumar and S. Sivanesan, Analyst, 2019, 144, 3111-3118.

32 L. Sijia, C. Jianan, W. Xia, Z. Xuan, H. Qi and H. Xiaohong, J. Hazard. Mater., 2019, 373, 408-416.

33 H. Yuling, H. Zelin, L. Jia and L. Gongke, Anal. Chem., 2013, 85, 6885-6893.

34 L. Huijun, L. Qingqing, H. Xinglei, Z. Ning, X. Zhouqing, W. Yan and W. Yuan, Cryst. Growth Des., 2018, 18(10), 6248-6256.

35 Z. Caihong, A. Lunhong and J. Jing, J. Mater. Chem. A, 2015, 3, 3074-3081.

36 A. Anand Babu Christus, P. Panneerselvam and A. Ravikumar, Anal. Methods, 2018, 10, 4378-4386.

37 Z. Zhiguang, L. Xinyong, L. Baojun, Z. Qidong and C. Guohua, RSC Adv., 2016, 6, 4289-4295.

38 L. Ai, C. Zhang, F. Liao, Y. Wang, M. Li, L. Meng and J. Jiang, J. Hazard. Mater., 2011, 198, 282-290.

39 S. K. Tama, J. Dusseault, S. Polizu, M. Menard, J.-P. Halle and L. H. Yahia, Biomater, 2005, 26, 6950-6961.

40 N. Wang, L. Zhu, D. Wang, M. Wang, Z. Lin and H. Tang, Ultrason. Sonochem., 2010, 17, 526-533.

41 H. Zhao, Y. Wang, Y. Wang, T. Cao and G. Zhao, Appl. Catal., $B, 2012,125,120-127$.

42 J. Su, M. Cao, L. Ren and C. Hu, J. Phys. Chem. C, 2011, 115, 14469-14477. 